\title{
'THERE IS AN OVERUSE OF IMPLANTS IN THE WORLD AND AN UNDERUSE OF TEETH AS TARGETS FOR TREATMENT'
}

Professor Jan Lindhe is an emeritus professor at the University of Gothenburg, where he was previously Chair of Periodontics and Dean of the School of Dentistry. Lindhe graduated from the Royal School of Dentistry in Malmö, Sweden. He is now one of the world's most renowned researchers in periodontology and is well known for his book Clinical periodontology and implant dentistry as well as hundreds of other publications. Jan Lindhe acted as editor of the Journal of Clinical Periodontology for over 30 years and continues to contribute to scientific debate and research.

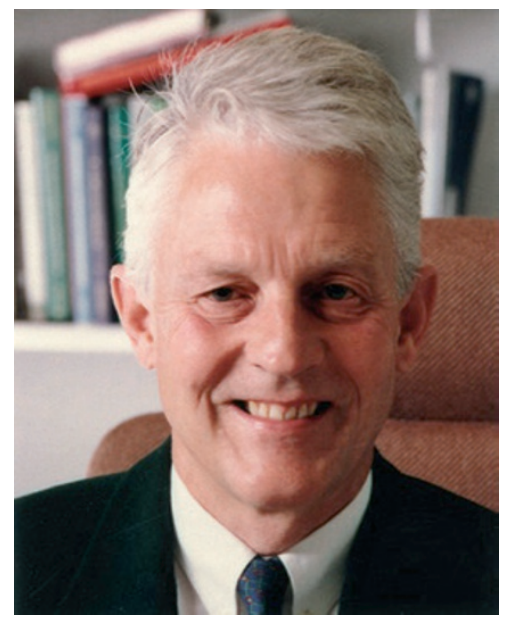

Research has linked periodontal disease to countless systemic illnesses. Is this the best way to stress the significance of good periodontal health to the public?

I believe there is some sort of association between periodontal disease and some general systemic conditions, but I don't think that should be the main motivation for maintaining good periodontal health. The initial motivation should be that gum disease is an infection. We try to manage infections all over the body, why should we ignore them in the oral cavity? It's not only periodontal disease that is infectious, as we know, caries is a bacterial disease. It's therefore logical to control the infection - not to eliminate it, because that is basically impossible - but to control it to the extent where the lesions you develop in your gum and hard tissue will as be minimal as possible.

\section{Should general dental practitioners (GDPs) play a more active role in screening for and preventing chronic inflammatory diseases outside of periodontitis?}

The GDP plays a very important role in screening patients for both caries and gum disease, and also plays a pivotal role in treating the disease as it occurs. Outside of the oral cavity, however, I'm not so sure. I think that the dentist's arena is pretty well described - it's intraoral and also maxillofacial in a sense but the dentist shouldn't be a pseudo-doctor for all types of disorders. A dentist may meet with patients more frequently
'We try to manage infections all over the body, why should we ignore them in the oral cavity?' than a physician, but the responsibility should not be with the dentist to diagnose diabetes or other inflammatory diseases. On the other hand, communication between the dentist and patient is often very open and consequently if the dentist suspects something is wrong, they may recommend their patient see a physician for further screening and diagnosis.

What is the most challenging periodontal problem facing clinicians today?

The most challenging problem is to control periodontal disease in most populations. We know gingivitis is the starting point for more advanced gum disease, but in the past we seem to have regarded gingivitis as a benign or uncomplicated inflammatory lesion in the mucosa or soft tissue. It has been shown that a long-standing inflammation in the gingiva may lead to attachment loss and may be a risk for tooth loss in the future. Long-standing gingivitis is the challenge for the profession to control because it can be done. More advanced disorders in the gums and unique forms of gum disease may be difficult to diagnose early but gingivitis can be diagnosed early and that is the responsibility of the dentist and dental hygienist together.

\section{What has been the most important breakthrough in periodontal research in the last $\mathbf{5 0}$ years?}

Well the most obvious breakthrough is that it has been established that bacteria are the cause of periodontal disease. Of course, this had been suspected a long time ago and there was indirect proof that this was the case. During the last 50 years it has been documented that if we don't allow bacteria to form plaque/biofilm on teeth, then gingival inflammation does not develop. In addition, if patients with advanced periodontal disease have their teeth cleaned and are kept in a very careful infection control programme, their disease can heal and disappear. This is crucial knowledge for the clinician. Another major breakthrough in clinical periodontology is studies from different microbiological laboratories identifying certain groups of bacteria as being more responsible than other groups of bacteria for gum disease.

You have spent a lot of time in Japan and were awarded the Order of the Rising Sun last year for your involvement in the Japanese dental care system. How does it compare to systems in Europe?

The Japanese dental care system is in many ways similar to the European system. It's founded on regular contact between the dentist and the patient, and the patient sees the dentist not only when he or she is in pain, but on a regular basis, so in that sense it's not so different. Together with our Japanese students, we performed clinical studies in Japan and documented that the Japanese are as eager as Swedish people to obtain and then maintain periodontal health. Levels of periodontal disease are not the same all over the world but in many industrialised countries they are. However, 
there are many obvious differences depending on tradition. In particular, in certain parts of the world people only go to the dentist when they have toothache and problems. But in our societies, in the Swedish society for sure, there is a formal regularity around seeing your dentist once every 6 months or year.

In addition, there is also an economical aspect to handle because it's not inexpensive to access dental care. Yet if you stop and think about how much people spend on their hair every year for example, people don't seem to hesitate to see the hairdressers and are happy to pay every second, third month, which all adds up. People don't think so much about how much they pay for beauty treatment in a year, but when it comes to dental care, a matter of health, it's something they seemingly want to escape. And of course in financial situations where you don't have money then one of the things you try to avoid is seeing the dentist, as instinctively you know that it costs money.

\section{You were Editor of the Journal of Clinical Periodontology for decades, how do you see dental publishing developing and how important is it?}

I think we will always need dental and medical journals in one form or another. The issue as I see it is with the printed journal. I'm an oldfashioned person and like to hold something in my hand and when I read I like to underline points to remember and make notes in the margin, but I don't think that the next generation will need that they are so used to studying on the Internet. I believe therefore that the number of printed journals will be reduced but the number of electronic journals will increase. I also believe this will importantly result in a shorter publication time, to disseminate knowledge more quickly, and that the scientific paper of the future will be constructed differently. I don't think you need a long introduction - I think you need materials and methods clearly described and results clearly described so that everyone can repeat them, and you need conclusions. I'm not so sure that you need a very extensive discussion section. With the introduction and the discussion shortened, the materials and methods, results and conclusion will be the integral sections of scientific papers. It's not that I think discussion is less important but it's often voluminous and the author describes to the audience what he or she believes that he or she has observed, which depends on training and what their experience is when they present their discussion. I believe much more in review papers that handle a number of different results and thus make the regular scientific paper shorter.

\section{What has been the proudest moment in your career?}

I don't know; that's a difficult question. I think my proudest moment was when I became a dentist. Another proud moment was when I was invited to give a talk for the first time, and that talk was in Norway, many, many years ago, but I remember that as a special and proud occasion. I was honoured and gratified to have the attention of a number of my colleagues and present my research findings.

\section{What role have you found most rewarding?}

The role of a teacher for graduate students. You always have quite a number of undergraduate students every semester, but you only have a handful of graduate students every year and that means that you can become very close to such students and play a pivotal role in their development.

\section{What would like to be remembered for?}

I don't know what I want to be remembered for, maybe that we published the Textbook of clinical periodontology and tried to base that book on scientific evidence rather than beliefs. I think it was one of the first books that really established scientific texts for graduate and undergraduate students that were based on research data rather than on one's own impressions. I remember in the good old days when you went on to a specialist programme in dentistry you always had an instructor or director of your undergraduate programme, who was a magnificent clinician but very often had little in terms of research data that could back up his concepts. This then meant that in that particular programme whatever the director said was 'the truth'. And then there could be a director of another programme, not so far away geographically, who had different ideas. If you have no research, no numbers, no figures, then it's just the opinion of someone that you teach. My textbooks Clinical periodontology and implant dentistry highlight that you should base the opinions you express to students on facts rather than your beliefs.

\section{Your recent research has focused heavily on implants. Has anything surprised you? Do we know enough about the long-term success of implants?}

We know first of all that implants work very well and in most patients, most implants function year after year without any problem. Then there are a few patients who lose their implants and why not? All spare parts that you place in the body are associated with some complications. To me it's rather impressive that dental implants have as good and as long a prognosis as they have. The drawback is of course that many dentists find it easier to remove a tooth and place an implant than to use the data in the literature to treat the tooth for its conditions. So maybe there is an overuse of implants in the world and an underuse of teeth as targets for treatment. But for obvious reasons dental implants have played and will play in the future a very important role in the rehabilitation of patients with tooth loss.

\section{How do you think future periodontologists should be trained?}

Future periodontists should be trained first and foremost to treat periodontal disease and associated issues, including aesthetic issues, but should also learn how to place implants and be careful in maintaining the soft and hard tissues around the implants.

They should be trained as traditional periodontists, but in addition be successful in their management of implants, which are becoming an increasing part of periodontology.

\section{Gum disease is known as the 'silent disease' - when will it be heard? At what point in the future do you think people will recognise its impact?}

Gum disease is a silent disease in the sense that you don't develop symptoms like toothache or a tooth or teeth starting to become mobile until you are very late in the disease process. The fact that it's a silent disease is something we cannot change. Yet it's up to the profession, up to the general dental practitioner, to use a periodontal probe when a patient comes for their regular check-up and identify what the problems are, if there are problems, and where they are located. If there is bleeding on probing then you know that there is something wrong and if there is a deepened pocket, there is either gingival inflammation or loss of attachment to the tissue around the tooth. Much of the public believe that a little bit of blood when you brush your teeth is healthy but that of course is not the case. I think the only way to make the silent disease heard is through the general dental practitioner, who can identify the problem, explain it to the patient and treat the problem.

INTERVIEW BY LAURA PACEY 